\title{
Social Media Analysis using Optimized K-Means Clustering
}

\author{
K. Madhuri1, Mr. K. Srinivasa Rao² \\ ${ }^{1}$ Associate Professor, ${ }^{2}$ Final M.Tech Student \\ ${ }^{1,2}$ Department of CSE, Sarada Institute of Science, \\ 1,2Technology and Management (SISTAM), Srikakulam, Andhra Pradesh, India
}

\begin{abstract}
Now a day's social media place an important role for sharing human social behaviors and participation of multi users in the network. The social media will create opportunity for study human social behavior to analyze large amount of data streams. In this social media one of the interesting problems is users will introduce some issues and discuss those issues in the social media. So that those discuss will contain positive or negative attitudes of each user in the social network. By taking those problems we can consider formal interpretation social media logs and also take the sharing of information that can spread person to person in the social media. Once the social media of user information is parsed in the network and identified relationship of network can be applied group of different types of data mining techniques.
\end{abstract}

However, the appropriate granularity of user communities and their behavior is hardly captured by existing methods. In this paper we are proposed optimized fuzzy means clustering algorithm for grouping related information. By implementing this algorithm we can get best group result and also reduce time complexity for generating cluster groups. The main goal of our proposed framework is twofold for overcome existing problems. By implementing our approach will be very scalable and optimized for real time clustering of social media.

\section{INTRODUCTION}

Clustering is the process of partitioning or grouping a given set of patterns into disjoint clusters. This is done such that patterns in the same cluster are alike and patterns belonging to two different clusters are different. Clustering has been a widely studied problem in a variety of application domains including neural networks, AI, and statistics. Data clustering is considered an interesting approach for finding similarities in data and putting similar data into groups. Clustering partitions a data set into several groups such that the similarity within a group is larger than that among groups. The idea of data grouping, or clustering, is simple in its nature and is close to the human way of thinking; whenever we are presented with a large amount of data, we usually tend to summarize this huge number of data into a small number of groups or categories in order to further facilitate its analysis. Moreover, most of the data collected in many problems seem to have some inherent properties that lend themselves to natural groupings. Nevertheless, finding these groupings or trying to categorize the data is not a simple task for humans unless the data is of low dimensionality (two or three dimensions at maximum.) This is why some methods in soft computing have been proposed to solve this kind of problem. Those methods are called "Data Clustering Methods" and they are the subject of this paper. Clustering algorithms are used extensively not only to organize and categorize data, but are also useful for data compression and model construction. By finding similarities in data, one can represent similar data with fewer symbols for example. Also if we can find groups of data, we can build a model of the problem based on those groupings.

As mentioned earlier, data clustering is concerned with the partitioning of a data set into several groups such that the similarity within a group is larger than that among groups. This implies that the data set to be partitioned has to have an inherent grouping to some extent; otherwise if the data is uniformly distributed, trying to find clusters of data will fail, or will lead to artificially introduced partitions. Another problem that may arise is the overlapping of data groups.

Overlapping groupings sometimes reduce the efficiency of the clustering method, and this reduction is proportional to the amount of overlap between groupings. Usually the techniques presented in this paper are used in conjunction with other sophisticated neural or fuzzy models. In particular, most of these techniques can be used as preprocessors for determining the initial locations for radial basis functions or fuzzy if then rules. The common approach of all the clustering techniques presented here is to find cluster centers that will represent each cluster. A cluster center is a way to tell where the heart of each cluster is located, so that later when presented with an input vector, the system can tell which cluster this vector belongs to by measuring a similarity metric between the input vector and al the cluster centers and determining which cluster is the nearest or most similar one. Some of the clustering techniques rely on knowing the number of clusters. In that case the algorithm tries to partition the data into the given number of clusters. K-means and Fuzzy C-means clustering are of that type. In other cases it is not necessary to have the number of clusters known from the beginning; instead the algorithm starts by finding the first large cluster, and then goes to find the second, and so on. however if the number of clusters is not known, K-means and Fuzzy C-means clustering cannot be used. Another aspect of clustering algorithms is their ability to be implemented in on-line or offline mode. On-line clustering is a process in which each input vector is used to update the cluster centers according to this vector position. The system in this case learns where the cluster centers are by introducing new input every time. In off-line mode, the system is presented with a training data set, which is used to find the cluster centers by analyzing all the input vectors in the training set. Once the cluster centers are found they are fixed, and they are used later to classify new input vectors. The techniques presented here are of the off-line type. A brief overview of the four techniques is presented here. Full detailed discussion will follow in the next section. 


\section{EXISTING SYSTEM:}

In the existing technique will take by performing clustering of social media information will face so many problems. For example take the $\mathrm{k}$ means clustering algorithm for performing clustering process will take more time and will not get efficient clustering result. By implementing the $\mathrm{k}$ means algorithm we can also face the problem of space complexity. In the $\mathrm{k}$ means algorithm we can also face the problems of random centroid selection. By choosing random centroids is poor generation of cluster groups. By overcome those problems we can implementing bisecting optimized cluster distance algorithm.

\section{PROPOSED SYSTEM:}

The amount of information shared on online social media has been growing during recent years. Muchcan be learned about the retail and finance behaviors of users by studying social media analysis. It is nothing new that retail companies market via social networks to discover what consumers think about branding, customer relationship management, and other strategies including risk prevention. A good example is the found correlation of data on Twitter with industry market behavior and sentiment posted by users. Social network analysis has a well-defined relation and background in sociology. With the rapid growth of the web forums and blogs, the user's participation on content creation led to a huge amount of dataset. Hence the advancement of data mining techniques is required. An overall discussion of one news forum called Slashdot, can be found in Social networks, it focus work like face pager. It is used to access data from social media like face book by using this data to develop a clustering framework using optimized fuzzy means cluster distance algorithm that is more accurate than existing methods. Clustering is used as an exploratory analysis tool that aims at categorizing objects into categories, so the association degree between the objects is maximal when belonging to the same categories. Clustering structures the data into a collection of objects that are similar or dissimilar and is considered an unsupervised learning. The application of our method is mainly on finding user groups based on activities and attitude features as suggested in the authority model.

The standard k-means algorithm takes extra time in calculating distance from each cluster's center in each iteration. The implementation process of $\mathrm{k}$ means algorithm is as follows.

1. Read the twitter data set from the twitter server.

2. Enter number of clusters to be performing and randomly choose the centroids from twitter dataset.

3. Take each data point (di) from dataset and calculate the Manhattan distance from data point to centroids' (ci). Distance $=$ (ci-di)

4. If check the closet distance of each centroid from the data point and that data points will be put into those clusters.

5. The step 3 and 4 will be repeated until there is no change in the centroids.

6. After completion of step 6 we can get group of clustered data.

7. The calculation of Manhattan distance we can also calculate each cluster sum squared error by $\mathrm{v}$ using following equation.

$\mathrm{SSE}=\Sigma \mathrm{i}=1 \mathrm{n}$ dis $(\mathrm{ci}, \mathrm{di})$
By implementing this algorithm will take time complexity and space complexity. This extra time can be saved by adapting this method. Implementation process of optimized fuzzy means clustering algorithm is as follows:

\section{Optimized Fuzzy Means Clustering Algorithm:}

The number of desired clusters, $\mathrm{k}$, and a dataset $\mathrm{D}=(\mathrm{d} 1, \mathrm{~d} 2 \ldots$ $\mathrm{dn}$ ) containing $\mathrm{n}$ data objects.

\section{Output:}

A set of k clusters.

Steps:

1. Randomly select $\mathrm{k}$ data objects from dataset $\mathrm{D}$ as initial clusters.

2. Calculate the matched words between each data object di $(1<=i<=n)$ and each cluster Center cj $(1<=\mathrm{j}<=\mathrm{k})$.

3. After completion of matched word we can find out sum squared error by using following formula.

SSE $=1 / w 2$

4. Calculate total number of words in a data point and centroid find out weight of each data points to centroid. The calculation of weight each twit is as follows. Weight (Wi) $=1 /$ dist (di, ci) $2 / \Sigma q=1 \mathrm{k} 1 /$ dist (ci, di)

5. After completion of weight of each data point to centroids check which data point is near by the centroids.

6. For every cluster center $\mathrm{cj}(1<=\mathrm{j}<=\mathrm{k})$, it compute the weight of data points $d(\mathrm{di}, \mathrm{cj})$ and assign the data objesct di to the nearest cluster.

Set cluster $[\mathrm{i}]=\mathrm{j}$;

Set $w[i]=d(d i, c j)$.

7. $i$ For each cluster center $\mathrm{j}(1<=\mathrm{j}<=\mathrm{k})$, recalculate the centers;

8. Until the center is same.

9. Output the clustering result.

\section{Operational Feasibility:}

Proposed projects are beneficial only if they can be turned out into information system. That will meet the organization's operating requirements. Operational feasibility aspects of the project are to be taken as an important part of the project implementation. Some of the important issues raised are to test the operational feasibility of a project includes the following: -

$>$ Is there sufficient support for the management from the users?

$>$ Will the system be used and work properly if it is being developed and implemented?

$>$ Will there be any resistance from the user that will undermine the possible application benefits?

This system is targeted to be in accordance with the abovementioned issues. Beforehand, the management issues and user requirements have been taken into consideration. So there is no question of resistance from the users that can undermine the possible application benefits.

The well-planned design would ensure the optimal utilization of the computer resources and would help in the improvement of performance status.

\section{SYSTEM DESIGN} UML Diagrams:

Systems design is the process or art of defining the architecture, components, modules, interfaces, and data for a 
system to satisfy specified requirements. One could see it as the application of systems theory to product development. There is some overlap with the disciplines of systems analysis, systems architecture and systems engineering If the broader topic of product development "blends the perspective of marketing, design, and manufacturing into a single approach to product development, then design is the act of taking the marketing information and creating the design of the product to be manufactured. Systems design is therefore the process of defining and developing systems to satisfy specified requirements of the user. Until the 1990s systems design had a crucial and respected role in the data processing industry. In the 1990s standardization of hardware and software resulted in the ability to build modular systems. The increasing importance of software running on generic platforms has enhanced the discipline of software engineering. Object-oriented analysis and design methods are becoming the most widely used methods for computer system design [citation needed]. The UML has become the standard language used in Object-oriented analysis and design [citation needed]. It is widely used for modeling software systems and is increasingly used for high designing non-software systems and organizations

\section{Use Case Relationships:}

Three relationships among use cases are used often in practice:

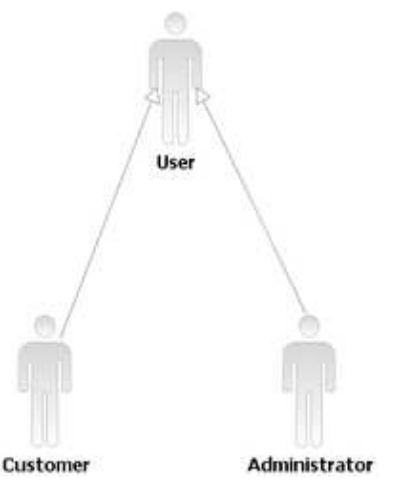

Fig 1 Diagram Building blocks

\section{Elements of a Collaboration Diagram}

A Collaboration diagram consists of the following elements:

\begin{tabular}{|l|l|}
\hline \multicolumn{1}{|c|}{ Element and its description } \\
\hline $\begin{array}{l}\text { Object: The objects interacting with each } \\
\text { other in the system. Depicted by a } \\
\text { rectangle with the name of the object in it, } \\
\text { preceded by a colon and underlined. }\end{array}$
\end{tabular}

\section{LANGUAGE SPECIFICATIONS}

\section{Java Technology}

Java Architecture: Java's architecture arises out of four distinct but interrelated technologies:

The Java programming language

The Java class file format

The Java Application Programming Interface

The Java virtual machine

When you write and run a Java program, you are tapping the power of these four technologies. You express the program in source files written in the Java programming language, compile the source to Java class files, and run the class files on a Java virtual machine. When you write your program, you access system resources (such as I/0, for example) by calling methods in the classes that implement the Java Application Programming Interface, or Java API. As your program runs, it fulfills your program's Java API calls by invoking methods in class files that implement the Java API. You can see the relationship between these four parts 
compile-time environment

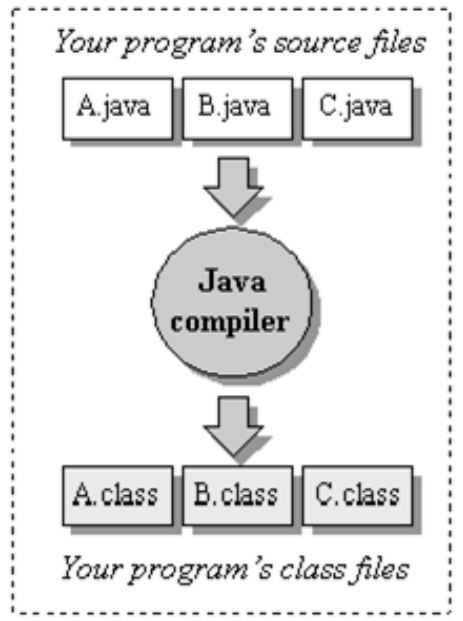

run-time environment

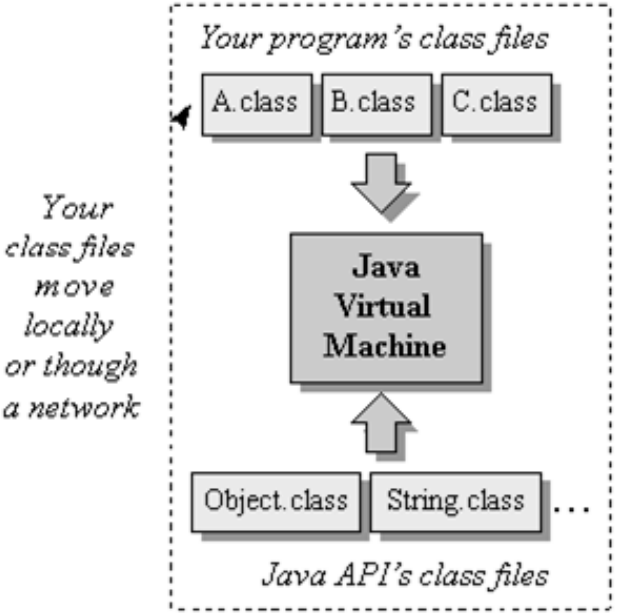

Fig 3 Java Programming Environment

Software Development Life Cycle

The Systems Development Life Cycle (SDLC), or Software Development Life Cycle in systems engineering, information systems and software engineering, is the process of creating or altering systems, and the models and methodologies that people use to develop these systems.

In software engineering the SDLC concept underpins many kinds of software development methodologies. These methodologies form the framework for planning and controlling the creation of an information system the software development process.

\section{WHAT IS SDLC}

A software cycle deals with various parts and phases from planning to testing and deploying software. All these activities are carried out in different ways, as per the needs. Each way is known as a Software Development Lifecycle MODEL (SDLC) [2].A software life cycle model is either a descriptive or prescriptive characterization of how software is or should be developed. A descriptive model describes the history of how a particular software system was developed. Descriptive models may be used as the basis for understanding and improving software development processes or for building empirically grounded prescriptive models.

\section{SDLC models:}

Development

$>$ The Linear model (Waterfall) - Separate and distinct phases of specification and development. - All activities in linear fashion. - Next phase starts only when first one is complete.

D Evolutionary development - Specification and development are interleaved (Spiral, incremental, prototype based, Rapid Application development). - Incremental Model (Waterfall in iteration), - RAD(Rapid Application Development) - Focus is on developing quality product in less time, - Spiral Model - We start from smaller module and keeps on building it like a spiral. It is also called Component based development.

$>$ Formal systems development - A mathematical system model is formally transformed to an implementation.

$>$ Agile Methods. - Inducing flexibility into development.

$>$ Reuse-based development - The system is assembled from existing components

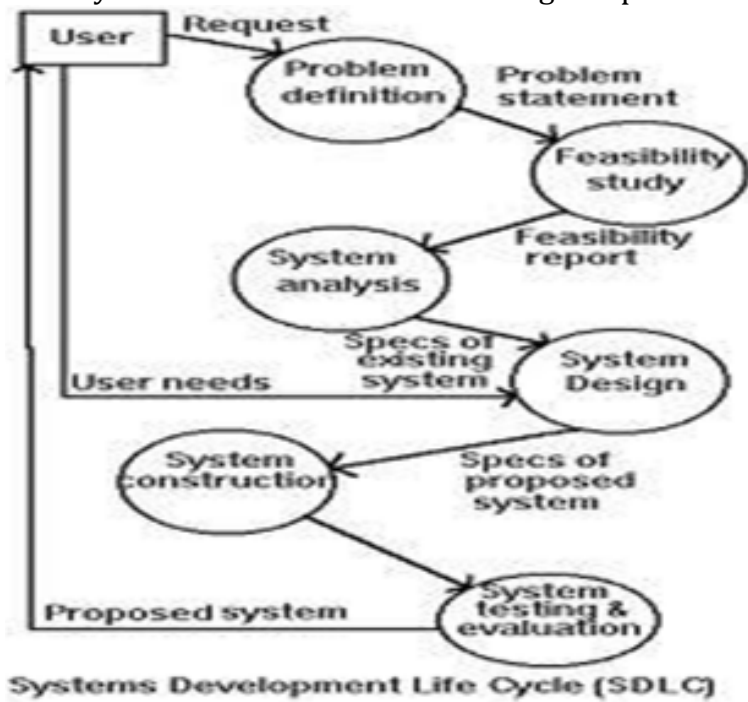

Fig 4 General life cycle model 


\section{Spiral Life Cycle Model:}

The spiral model is similar to the incremental model, with more emphases placed on risk analysis. The spiral model has four phases: Planning, Risk Analysis, Engineering and Evaluation. A software project repeatedly passes through these phase in iteration (called Spirals in this model). The baseline spirals, starting in the planning phase, requirements are gathered and risk is assessed. Each subsequent spiral builds on the baseline spiral. Requirements are gathered during the planning phase. In the risk analysis phase, a process is undertaken to identify risk and alternate solutions. A prototype is produced at the end of the risk analysis phase. Software is produced in the engineering phase, along with testing at the end of the phase. The evaluation phase allows the customer to evaluate the output of the project to date before the project continues to the next spiral. In the spiral model, the angular component represents progress, and the radius of the spiral represents cost.

\section{TESTING}

Testing methods:

The box approach:

Software testing methods are traditionally divided into white- and black-box testing. These two approaches are used to describe the point of view that a test engineer takes when designing test cases

\section{White box testing:}

White box testing is when the tester has access to the internal data structures and algorithms including the code that implement these.

\section{Types of white box testing:}

The following types of white box testing exist:

$>$ API testing (application programming interface) testing of the application using public and private APIs

$>$ Code coverage - creating tests to satisfy some criteria of code coverage (e.g., the test designer can create tests to cause all statements in the program to be executed at least once)

$>$ Fault injection methods - improving the coverage of a test by introducing faults to test code paths

$>$ Mutation testing methods

$>$ Static testing - White box testing includes all static testing

\section{Black box testing:}

Black box testing treats the software as a "black box"without any knowledge of internal implementation. Black box testing methods include: equivalence partitioning, boundary value analysis, all-pairs testing, fuzz testing, model-based testing, traceability matrix, exploratory testing and specification-based testing. Specification-based testing: Specification-based testing aims to test the functionality of software according to the applicable requirements.] Thus, the tester inputs data into, and only sees the output from, the test object. This level of testing usually requires thorough test cases to be provided to the tester, who then can simply verify that for a given input, the output value (or behavior), either "is" or "is not" the same as the expected value specified in the test case.

Specification-based testing is necessary, but it is insufficient to guard against certain risks.

\section{CONCLUSION}

This paper we are proposed an efficient clustering algorithm for reduce the time complexity and space complexity. This paper proposes optimized fuzzy means clustering algorithm for getting better cluster result in data set. By implementing this process we can easily find out similar data object in data set by calculating weight of each data object to centroids. The calculation of weight of data object will repeat until the no changes occur in the centroids. By applying this process we can reduce number of iteration compared to existing algorithm of k means. So that each data point from each cluster center in each iteration due to which running time of algorithm is saved. By implementing proposed system we can efficiently improve speed of the clustering and accuracy by reducing the computational complexity of standard kmeans algorithm.

\section{REFERENCES}

[1] Andreas M Kaplan and Michael heilien. Users of the world, unite! the challenges and opportunities of social media. Business horizons, 53(1):59-68, 2010.

[2] BogdanBatrinca and Philip C Treleaven. Social media analytics: a survey of techniques, tools and platforms. AI \& SOCIETY, 30(1):89- 116, 2015.

[3] David Lazer, Alex Sandy Pentland, LadaAdamic, Sinan Aral, Albert Laszlo Barabasi, Devon Brewer, Nicholas Christakis, Noshir Contractor, James Fowler, Myron Gutmann, et al. Life in the network: the coming age of computational social science. Science (New York, NY), 323(5915):721, 2009.

[4] Claudio Cioffi-Revilla. Computational social science. Wiley Interdisciplinary Reviews: Computational Statistics, 2(3):259-271, 2010.

[5] HaewoonKwak, Changchun Lee, Hosung Park, and Sue Moon. What is twitter, a social network or anews media? In Proceedings of the 19th international conference on World Wide Web, pages 591-600. ACM, 2010.

[6] Michael D Conover, Clayton Davis, Emilio Ferrara, KarissaMcKelvey, FilippoMenczer, and Alessandro Flammini. The geospatial characteristics of a social movemen communication network.Plos one, 8(3):e55957, 2013.

[7] Bruce A. Maxwell, Frederic L. Pryor, Casey Smith, "Cluster analysis in cross-cultural research" World Cultures 13(1): 22-38, 2002.

[8] KiriWagstaff and Claire Cardie Department of computer science, Cornell University, USA “Constrained k- means algorithm with background knowledge".

[9] Thomas H. Cormen, Charles E. Leiserson, and Ronald L. Rivest, Introduction to Algorithms, Prentice Hall, 1990.

[10] Anil K. Jain, M. N. Murty, P. J. Flynn, "Data Clustering: A Review," ACM Computing Surveys, 31(3): 264-323 (1999).

[11] BogdanBatrinca and Philip C Treleaven. Social media analytics: a survey of techniques, tools and platforms. AI \& SOCIETY, 30(1):89- 116, 2015.

[12] Emilio Ferrara, Mohsen JafariAsbagh, OnurVarol, VahedQazvinian, FilippoMenczer, and Alessandro Flammini. Clustering memes in social media. In Proceedings of the 2013 IEEE/ACM International Conference on Advances in Social Networks Analysis and Mining, pages 548-555. IEEE, 2013. 